\title{
ON A CERTAIN TRANSPORT SCHEDULING PROBLEM FOR HETEROGENEOUS BUS FLEET
}

In this paper we consider a certain transport scheduling problem for heterogeneous bus fleet. We suppose that some restrictions are given for sets of vehicles and trips. We study some special cases of this problem that can be solved in polynomial time.

\section{Introduction}

We deal with the problem of finding an admissible transport schedule for heterogeneous bus fleet. We suppose that the place of departure and arrival is the same for every trip and the set of vehicles that can perform a given trip is restricted. This problem was studied in [2] and its special case (for two types of vehicles) in [3] Its complexity is still an open question. It is known, that the generalisation of this problem (where the places of departure and arrival can be different) is NP-hard.

Let $V=\left\{v_{1}, \ldots, v_{m}\right\}$ be the set of vehicles and $S=\left\{s_{1}, \ldots, s_{n}\right\}$ be the set of trips. Every trip $s_{i}=\left(t_{i}^{D}, t_{i}^{A}, L_{i}\right), 0 \leq t_{i}^{D}<t_{i}^{A}$ is determined by the time of departure $t_{i}^{D}$, time of arrival $t_{i}^{A}$ and by the list of vehicles $L_{i} \subseteq V$ that can perform the trip $s_{i}$. We suppose that

1. the place of departure and arrival is the same for every trip,

2. $t_{i}^{X} \neq t_{j}^{Y}$ for all $i, j \in\{1, \ldots, n\}, i \neq j$ and $X, Y \in\{D, A\}$

3. $\forall s_{i}, s_{j} \in S:\left(i<j \Leftrightarrow \mathrm{t}_{\mathrm{i}}^{\mathrm{D}}<t_{j}^{D}\right)$,

4. the trip $s_{i}$ precedes the trip $s_{j}\left(s_{i}<s_{j}\right)$ if $i<j$ and $t_{i}^{A}<t_{j}^{D}$.

From the condition (4), it is easy to show that $(S, \prec)$ is a partially ordered set with the relation " $<$ ". More about partially ordered sets (posets) can be found in [8].

We say that the trips $\forall s_{i}, s_{j} \in S$ are of the same type if $L_{i}=$ $=L_{j}$. An admissible running board of the vehicle $v_{i}$ is the chain $T_{i}=S_{i_{1}}<S_{i_{2}}<\ldots<S_{i_{k}}$ where vi $\in L_{i_{1}} \cap L_{i_{2}} \cap \ldots \cap L_{i_{k}}$.

A schedule $R=\left(T_{1}, \ldots, T_{m}\right)$ is admissible if $T_{i}$ is admissible running board of the vehicle $v_{i}$ (for $i=1, \ldots, m$ ), $T_{1} \cup T_{2} \cup \ldots \cup$ $T_{m}=S$ and $T_{i} \cap T_{j}=\varnothing(i, j \in\{\forall 1,2, \ldots, m\}$. A set of all admissible schedules will be denoted by $\Re$. We will deal with the decision problem, whether $\Re=\varnothing$ or $\Re \neq \varnothing$.

\section{Mathematical models}

In this section we present two models of the mentioned decision problem. The first is the model based on a bivalent programming formulation.

By ordering increasingly the times of departures and arrivals, we obtain the vector

$$
\vec{t}=\left(t_{1}^{D}, \ldots, t_{1}^{A}, \ldots, t_{i}^{D}, \ldots, t_{i}^{A}\right)=\left(t_{1}, t_{2}, \ldots t_{2 n}\right)
$$

where $t_{k}<t_{l} \Leftrightarrow k<l$. Let $K=\{1,2, \ldots, 2 n\}$ be the set of the indices of the times in the vector $\vec{t}$. Let $I=\{1,2, \ldots, m\}$, be the index set of the vehicles and $J=\{1,2, \ldots, n\}$ be the indices of the trips.

Let $x_{i j}$ be a decision variable with the value $x_{i j}=1$ if $s_{j}$ is performed by $v_{i}$ and $x_{i j}=0$ otherwise. We need to decide if there exists a matrix $X=\left(x_{i j}\right) m \times n$ for which the following constraints hold:

$$
\sum_{i \in I} a_{i j} x_{i j}=1 \quad j \in J
$$

$\sum_{j \in J} a_{i j} b_{k j} x_{i j} \leq 1 \quad i \in I, k \in K$

$x_{i j} \in\{0,1\} \quad i \in I, j \in J$

where

$a_{i j}= \begin{cases}1 & \text { if } v_{i} \in L_{j} \\ 0 & \text { if } v_{i} \notin L_{j}\end{cases}$

$b_{k j}= \begin{cases}1 & \text { if } t_{j}^{D} \leq t_{k} \leq t_{j}^{A} \\ 0 & \text { if otherwise }\end{cases}$

\footnotetext{
* Peter Czimmermann

Faculty of Management and Informatics, University of Žilina, Slovak Republic, E-mail: petocimo@frcatel.fri.utc.sk
} 
Conditions (1) ensure that every trip $s_{j}$ is assigned to exactly one vehicle (for which $v_{i} \in L_{j}$ ).

Conditions (2) ensure that the vehicle can perform at most one trip in every moment. The existence of some polynomial method for finding the matrix $X$ is still an open question.

The model based on graph theory.

Definition 1. (see [6]) Let $G=(V, E)$ be a graph and $\mathrm{C} \neq \varnothing$ be a set of colors.

A (vertex) coloring of $G$ is a function $c: V \rightarrow C$ such that subgraphs induced by each color class have no edges.

Definition 2. (see [5]) Let the sets $L v \subseteq C$ be assigned to each vertex $v \in V$. We call the vertex coloring $c: V \rightarrow C$ of $G$ with property $\forall v \in V c(v) \in L_{v}$ a coloring from the lists (or list coloring).

Definition 3. (see [1]) A conditional coloring of $G$ with respect to a graphical property $\mathfrak{B}$ is an assignment of colors to its vertices so that subgraphs induced by each color class satisfy the property is.

Since $(S,<)$ is the poset, we can define a transitive digraph $G_{S}=\left(V_{S}, E_{S}\right)$ where $V_{S}=S$ and $\forall s_{i}, s_{j} \in S s_{j}\left(s_{i}, s_{j}\right) \in E_{S} \Leftrightarrow$ $s_{i}<s_{j}$. The problem of finding the admissible schedule can be solved as the problem of finding some conditional coloring of $G_{S}$ from the lists which satisfies the property $P_{o p}$ : vertices of the same color form an oriented path (or a transitive tournament) in $G_{S}$. Every color corresponds to one vehicle. For every vertex (trip), the list of admissible colors (the set of vehicles that can perform this trip) is specified. Vertices of the same color form a chain in $(S, \prec)$ which corresponds to some admissible running board.

The existence of polynomial algorithm for conditional coloring from the lists with respect to the property that vertices of the same color form an oriented path in $G_{S}$ is an unsolved problem.

\section{Cases that can be solved in polynomial time}

In this section we study some special cases that can be solved in polynomial time. We prefer a graph theoretical approach.

1. Let $\left|L_{1}\right|=\cdots=\left|L_{n}\right|=1$. This case is trivial, every vertex $v_{t}$ $\in G_{S}$ is colored by unique color from the list $L_{i}$. There is an admissible schedule if and only if the vertices of the same color form an oriented path in $G_{S}$. We are able to verify this in polynomial time.

2. Let $\left|L_{1}\right|=\cdots=\left|L_{n}\right|=2$. Since $G_{S}$ is transitive, vertices of every oriented path form also a transitive tournament in $G_{S}$. Let $G=(V, E)$ be a graph for which $V=V_{S}$ and the edge $\left\{s_{i}\right.$, $\left.s_{j}\right] \in E$ if and only if the trips $s_{i}, s_{j}$ are incomparable by relation $<$. It is easy to show that there is a coloring of $G$ from the lists $L_{1}=L_{2}=\cdots=L_{n}$ if and only if there exists a conditional coloring of $G_{S}$ from the lists with respect to the property $P_{o p}$. It is known (see also [7]) that the list coloring of the graph is solvable in polynomial time if every list contains exactly two elements (colors).

3. If $L_{1}=L_{2}=\cdots=L_{n} \subseteq V$ then we are able to transform this case to the problem of covering a poset by disjoint union of chains - the solution of this problem is based on the famous Dilwort's Chain Decomposition Theorem [4] and we are able to find it in polynomial time.

4. Let $L_{i} \cap L_{j}=\varnothing$ or $L_{n} \subseteq V$ for all $i, j\{1, \ldots, n\}$. We can decompose $(S,<)$ into subposets $\left(S_{1},<\right), \ldots,\left(S_{k},<\right)$ (see [8]) where $S_{i} \cap S_{j}=\varnothing, S_{1} \cup \cdots \cup S_{k}=S$, and two trips $s_{i}$, $s_{j}$ belong to the same subposet if $L_{i}=L_{j}$. We can solve this case for every poset $\left(S_{i},<\right)$ separately as in (3).

Acknowledgement: The research was supported by the Slovak Scientific Agency, Grant No.1/0490/03.

\section{References}

[1] Chartrand, G., Geller, D. P., Hedetniemi, S. T.: A Generalization of the Chromatic Number, Proc. Camb. Philos. Soc. 64:265-271, 1968

[2] CZIMMERMANN, P.: A Note on Bus Schedules with Several Types of Vehicles, Journal of Information, Control and Management Systems, vol. 2, No. 2/2003

[3] CZIMMERMANN, P., PEŠKO, S.: The Bus Schedules with Two Types of Vehicles, 3rd International Conference APLIMAT, 2004

[4] DILWORTH, R. P.: A Decomposition Theorem for Partially Ordered Sets, Ann. of Math. 51, 161-166, 1950

[5] DIESTEL, M.: Graph Theory, Springer-Verlag, New York, 1997

[6] HARARY, F.: Graph Theory, Reading(Mass.), Addison-Wesley, 1969

[7] PEŠKO, S.: Optimization of NP-hard transport schedules (in Slovak), Habilitation Thesis, University of Zilina, 2002

[8] Schröder, B. S. W.: Ordered Sets, Birkhäuser, Boston, 2003. 